\title{
Surface-Pressure Measurements of Second-Mode Instability in Quiet Hypersonic Flow
}

\author{
Malte Estorf* and Rolf Radespiel ${ }^{\dagger}$ \\ Technical University Braunschweig, 38106 Braunschweig, Germany \\ Steven P. Schneider ${ }^{\ddagger}$ \\ Purdue University, West Lafayette, IN, 47097-1282, USA \\ Heath B. Johnson ${ }^{\S}$ \\ University of Minnesota, Minneapolis, MN, 55455, USA \\ Stefan Hein \\ DLR - Institute of Aerodynamics and Flow Technology, 37073 Göttingen, Germany
}

\begin{abstract}
Surface pressure-sensors have been used to measure the second-mode boundary layer instability on a $7^{\circ}$ half-angle sharp cone at zero angle of attack in the quiet Mach-6 wind tunnel at Purdue and in the conventional Mach-6 wind tunnel in Braunschweig. The measurements were made using a stream-wise array of high-frequency sensors. They show the second-mode waves in quiet and noisy flow at different unit Reynolds numbers. The quiet flow data is compared to results in noisy flow. The signal quality allows for the calculation of amplification rates, which are compared to the results of linear stability computations.
\end{abstract}

\section{Nomenclature}

Latin symbols

a sonic speed

A flow area

$f \quad$ frequency

$l \quad$ tube length

$M \quad$ Mach number

$P \quad$ tube pressure

$\hat{p}(f) \quad$ pressure fluctuation amplitude

$R \quad$ mass specific gas constant

Re Reynolds number

$s \quad$ arc length along surface

$t \quad$ time

$T$ temperature

$u$ flow velocity in tube
$U \quad$ flow velocity in test section

$x \quad$ axial position

Greek symbols

$-\alpha_{i} \quad$ log. growth rate per arc length

$\gamma \quad$ ratio of specific heats

$\rho \quad$ fluid density

Indices

$0 \quad$ initial quantity

$\infty \quad$ free-stream quantity

$e \quad$ edge quantity

$t$ tube, stagnation quantity

* $\quad$ sonic condition

* Research Assistant, Member AIAA

${ }^{\dagger}$ Professor, Senior Member AIAA

$\ddagger$ Professor, Associate Fellow AIAA

§Senior Research Associate, Senior Member AIAA

『Research Scientist 


\section{Introduction}

Much of the uncertainty in the aerothermodynamic design of hypersonic flight vehicles is due to the poor understanding and uncertain prediction of laminar to turbulent boundary layer transition. The transition process is associated with the growth and final breakdown of disturbances in the boundary layer flow. Several instability modes are known. The disturbances they generate have been detected by a multitude of experimental techniques and predicted in numerical investigations. Schlichting and Gersten ${ }^{1}$ and Schneider ${ }^{2}$ provide comprehensive lists of literature on the subject. However, the mechanism-based prediction of transition in hypersonic flow is an outstanding and demanding task. ${ }^{2}$ In particular, it is difficult to obtain experimental data at well-controlled conditions. Among the multitude of unspecified influences on hypersonic transition, the most well known is the acoustic noise that is radiated from the turbulent boundary layer on the nozzle wall in conventional hypersonic wind tunnels. ${ }^{3,4}$ Therefore the comparison of instability measurements in noisy and quiet hypersonic flow is of particular interest. In symmetric flow at hypersonic speeds the dominant instability is the second mode. ${ }^{5}$ Some measurements of second mode waves have been carried out in the past with hot wires in both noisy and quiet flow. ${ }^{2}$ However, these measurements are very difficult due to the limited mechanical strength of small hot wires with sufficiently high frequency response. Moreover, the downstream influence of the hot wire's support precludes simultaneous amplitude measurements at several stream-wise positions. Hence, an appropriate non-intrusive measurement technique is desirable. Recently Fujii ${ }^{6}$ has shown that fast surface pressure sensors can measure the second mode on a cone in noisy hypersonic flow. In the present work this technique is used to measure the second mode under quiet flow conditions in the Boeing/AFOSR Mach-6 quiet tunnel of Purdue University. Those measurements are compared to measurements under noisy conditions in the Purdue tunnel and in the Mach-6 hypersonic facility at Technical University Braunschweig.

\section{Experimental Setup}

\section{II.A. The Boeing/AFOSR Mach-6 Quiet Tunnel}

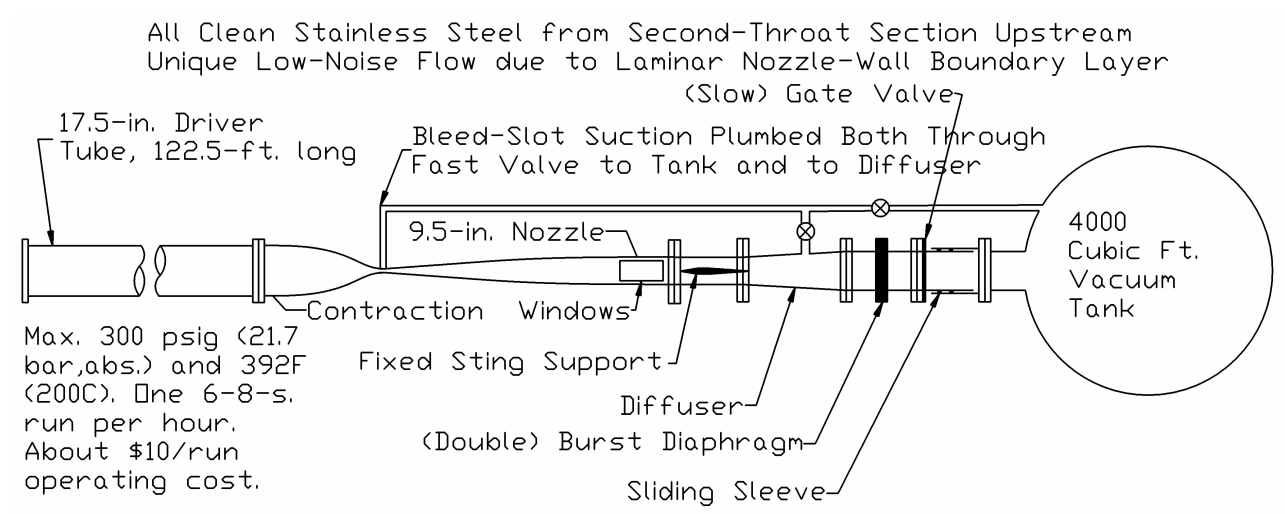

Figure 1. Schematic of the Boeing/AFOSR Mach-6 Quiet Tunnel

The Boeing/AFOSR Mach-6 quiet tunnel at Purdue University is a blowdown facility designed as a Ludwieg tube as sketched in Fig. 1. The $37.4 \mathrm{~m}$ long heated driver tube on the left serves as a pressurized air reservoir which discharges through the converging-diverging nozzle into the vacuum tank. Before each run the pressurized assembly is separated from the vacuum tank by a pair of diaphragms placed downstream of the test section. When the diaphragms burst, an expansion wave travels upstream through the test section into the driver tube. There it reflects forth and back and changes the state of the air each time it passes. This causes the flow condition in the test section to change approximately every $200 \mathrm{~ms}$ when the wave reaches the nozzle. Fig. 2 shows the stepwise change of pressure as measured at the contraction wall ${ }^{7}$. Fig. 2 also shows measurements from a Pitot probe in the test section. The Pitot pressure shows the sudden change from noisy to quiet flow condition when the contraction pressure falls below a certain pressure limit (here $\approx 145$ psia). The quiet flow is achieved only by extensive care in design and construction including a large driver tube and a highly polished nozzle with bleed slots for the contraction wall boundary layer. ${ }^{8}$ Although 


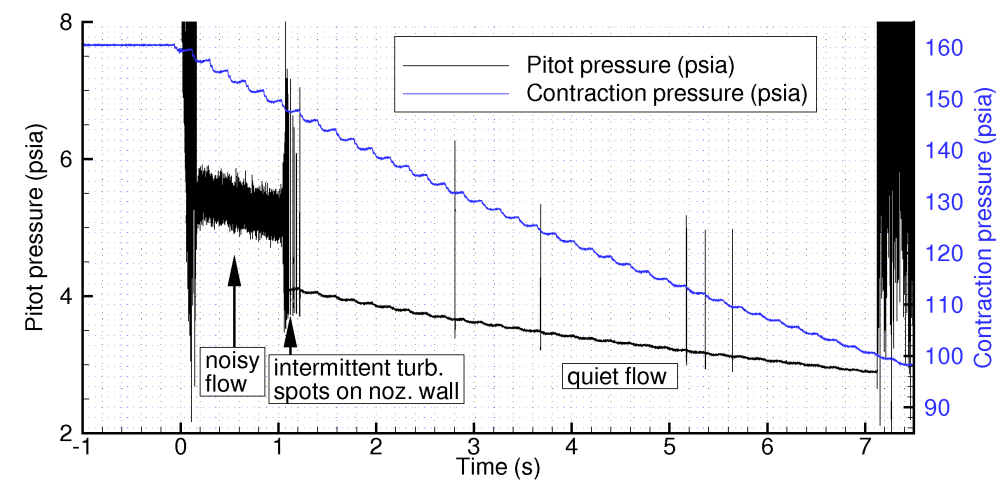

Figure 2. Sample of contraction pressure and Pitot pressure in Boeing/AFOSR Mach-6 Quiet Tunnel

the quiet performance degraded in late spring $2007,{ }^{9}$ it was restored in the late summer by repolishing the throat. ${ }^{10}$ When the measurements presented in this paper were made, the tunnel was running quiet up to stagnation pressures of 145 psia. By closing the bleeds for boundary layer suction at the nozzle throat the tunnel can be operated in noisy mode with turbulent nozzle-wall boundary layers. The tunnel can be operated at any pressure up to 270 psia.

\section{II.B. The Hypersonic Ludwieg Tube Braunschweig}

A schematic of the hypersonic Ludwieg tube Braunschweig (HLB) is given in Fig. 3. In this facility the driver tube is separated from the low pressure section by a fast-acting valve. Only the first $3 \mathrm{~m}$ long section of the driver tube is heated which suits the amount of gas that escapes during one run. The valve consists of a streamlined body on the tube axis with a pneumatically driven piston that plugs into the nozzle throat. The valve opens within $20 \mathrm{~ms}$ and closes after the expansion wave has passed back and forth within the tube; that is, about $80 \mathrm{~ms}$ after full opening. A detailed numerical analysis has been made for the onset of flow and the establishment of steady flow conditions in the test section. ${ }^{11}$ The nozzle maintains an opening half angle of $3^{\circ}$ which results in slightly expanding flow in the test section with Mach numbers between 5.8 and 5.95 depending on the axial position and on the unit Reynolds number. The latter can be chosen by the initial driver tube pressure between $3 \times 10^{6} / \mathrm{m}$ and $20 \times 10^{6} / \mathrm{m}$. More details on the HLB were reported earlier. ${ }^{12,13}$

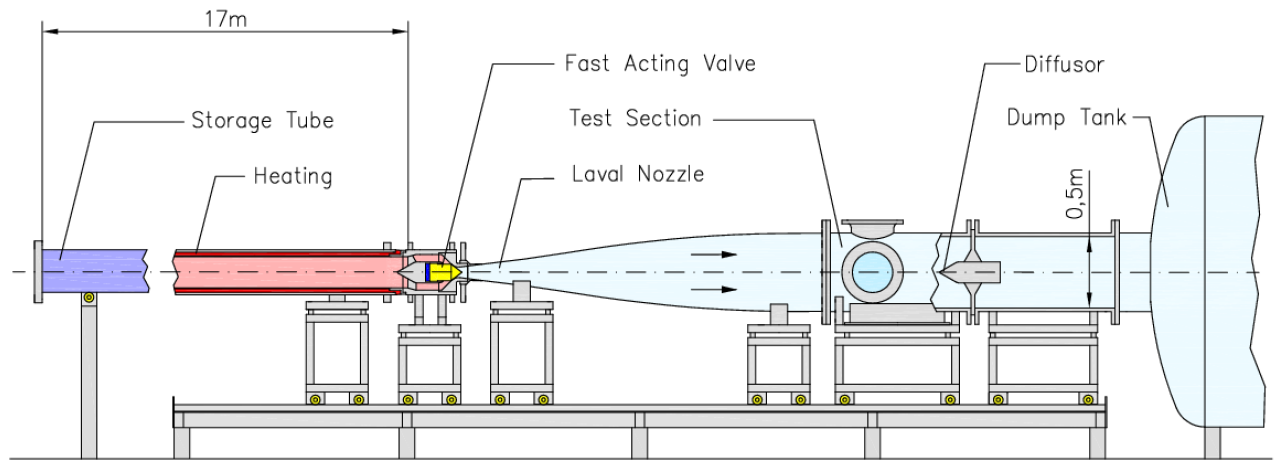

Figure 3. Schematic of the hypersonic Ludwieg tube Braunschweig

\section{II.C. Model and Instrumentation}

Measurements in both facilities were made on the same $7^{\circ}$ sharp cone of $495 \mathrm{~mm}$ length. The model was made from Plexiglas to allow future infrared heat transfer measurements on the same cone. The apex was made from steel in order to provide better mechanical strength. The accuracy of the tip was visually checked under a microscope. The nose radius was measured with a stage micrometer as about $0.01 \pm 0.003 \mathrm{~mm}$. The model was instrumented with a stream-wise array of high-frequency pressure sensors of type PCB M131A32. The 
sensors were flush mounted in the model surface at three axial positions ( $x=340 \mathrm{~mm}, 365 \mathrm{~mm}, 0.390 \mathrm{~mm})$ for the experiments in Purdue and at four positions ( $x=315 \mathrm{~mm}, 340 \mathrm{~mm}, 365 \mathrm{~mm}, 390 \mathrm{~mm})$ for the experiments in Braunschweig. The diameter of the sensing area of those sensors is $3.18 \mathrm{~mm}$. Power was supplied to the pressure sensors using an instrument supplied by the manufacturer (PCB 482A22), which at the same time also performed signal conditioning. According to the manufacturer's specification the resonance frequency of the pressure sensors is larger than $1 \mathrm{MHz}$ and the output signal is high-pass filtered at $10 \mathrm{kHz}$. The sensors are calibrated in a shock tube by the manufacturer and have sensitivities between 150 and $167 \mathrm{mV} / \mathrm{psi}$. For details on the the measurement technology the reader is referred to the manufacturer's website. ${ }^{14}$

\section{II.D. Data Acquisition}

The data acquisition in Purdue was performed with an oscilloscope (Tektronix DPO 7054) at a sampling rate of $4 \mathrm{MS} / \mathrm{s}$. The voltage range was chosen as $\pm 5 \mathrm{mV}$ for runs with quiet conditions and $\pm 25 \mathrm{mV}$ for noisy runs. The oscilloscope was operated in a "High-Res" mode where it samples the data internally at the maximum sampling rate and averages the data on the fly into memory. According to the manufacturer this mode gives an effective resolution of 11 bits at $4 \mathrm{MS} / \mathrm{s}$ (compared to 8 bits without averaging) and at the same time filters high frequency noise. In this mode the oscilloscope writes the data in a 16-bit format (2 bytes). According to the sensor calibration this gives an output resolution of $10^{-6} \mathrm{psi}$ using the quiet mode settings. The scope has 50 megabytes per channel of memory.

In Braunschweig a 16 bit transient recorder PCI-express card (Spectrum M2i.4652) was used for data acquisition with a PC. The card allowed a maximum sampling rate of $3 \mathrm{MS} / \mathrm{s}$. The amplification of the card was set to measure a voltage range of $\pm 50 \mathrm{mV}$.

\section{II.E. Model Installation}

In the Purdue quiet tunnel the base of the cone was installed $71 \mathrm{~mm}$ upstream from the end of the nozzle on the axis of the tunnel. Due to the tight fabrication tolerances of the sting support section and the snug sliding fit of the sting itself, the cone was assumed to be well aligned with the nozzle axis. Hence, the angle of attack was not checked before the experiments. After the test series a single measurement was taken with the cone model turned by $180^{\circ}$ around its base support plate. The flow condition in that run was noisy and evaluation showed later that the boundary layer was already transitional at the sensors. However, the maximum 2nd mode frequency in that run was about $10 \mathrm{kHz}$ smaller than in a run at identical conditions with the model turned by $180^{\circ}$ (not shown in this paper). This is likely due to a small angle of attack with the sensor array being slightly windward in all measurements presented in this paper at quiet conditions.

For the Braunschweig measurements the cone was installed with its apex $50 \mathrm{~mm}$ upstream from the beginning of the test section. The cone axis was positioned $50 \mathrm{~mm}$ above the axis of the tunnel in order to avoid a conical compression wave that is known to focus on the axis within the test section. ${ }^{15}$ The cone was aligned to the flow by measuring the line of transition with an infrared camera. The alignment was improved in subsequent tunnel runs until the transition line was found to be straight on the cone to within $\pm 10 \mathrm{~mm}$ from two perpendicular view angles.

\section{Determination of flow conditions}

\section{III.A. Purdue experiments}

The driver-tube pressure was measured at the entrance of the contraction during all tunnel runs. A temperature measurement was taken with a thermocouple at the end of the driver tube before every run. However, the temperature within the tube is stratified and there are also local differences in the tube temperature due to nonuniform heating and insulation. ${ }^{16}$ To improve the estimate of the mean temperature in the tube the trace of the pressure was used to find the temperature-dependent velocity of sound that corresponds to the frequency of the expansion-wave reflections within the tube. For this the flow of the air out of the tube was modeled by two characteristic lines corresponding to the head and the tail of the expansion wave as shown in Fig. 4. The details of the calculation are given in the appendix. A formula for the temperature at $X=0$ after $n$ reflections of the wave is given there as Eq. (7). The traveling times of the head and tail of the wave are given in Eqs. (10) and (11), respectively, as functions of the tube Mach number $M_{t}$.

Applying the isentropic relation $P / P_{0}=\left(T / T_{0}\right)^{\gamma / \gamma-1}$ to Eq. (7) shows that the pressure ratio between 


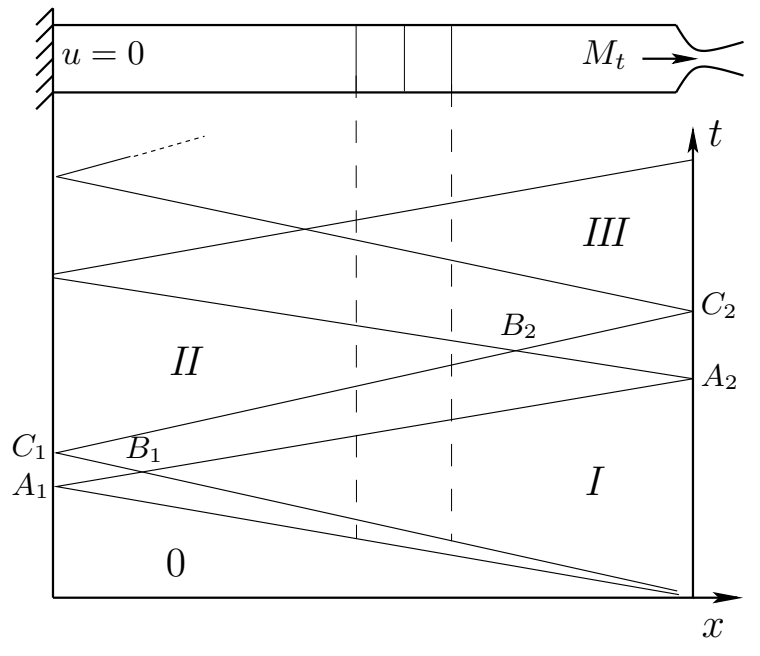

(a) characteristic diagram

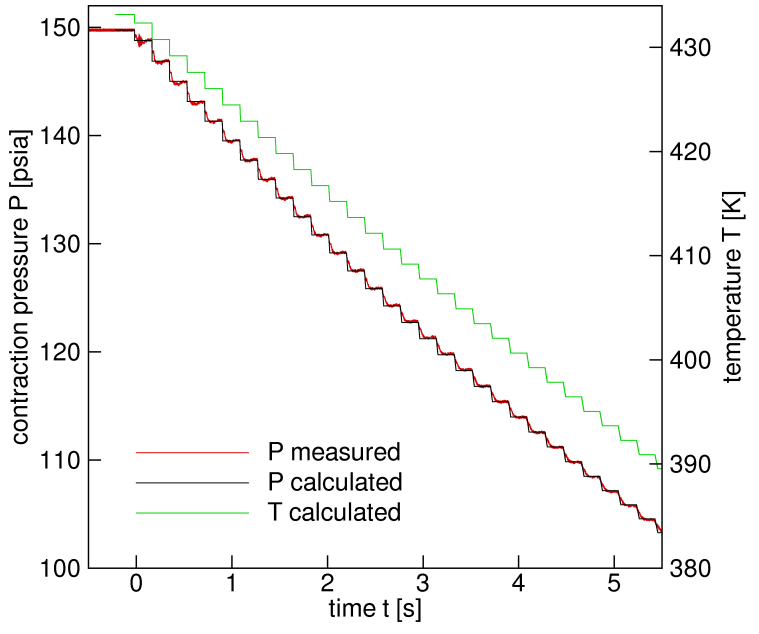

(b) sample evaluation

Figure 4. Illustration of driver tube gas condition modeling for Purdue quiet tunnel

subsequent wave reflections is only a function of the tube Mach number. Hence, with the measured pressure trace the tube Mach number can be estimated to fit the height of the pressure steps. Finally, the length of the steps can be fitted to the measured trace by adjusting the estimate of the initial mean temperature in the tube. These two estimates have been performed for all tunnel runs.

Figure 4b shows a pressure trace recorded during a quiet run with initial pressure of 149.8 psia and the corresponding calculations for the pressure and the temperature. The tube Mach number was $M_{t}=0.0046$ during the first $2.7 \mathrm{~s}$ and changed to $M_{t}=0.0044$ after that period. The initial temperature of the gas assumed for the calculations was $T_{0}=434 \mathrm{~K}$ which agrees with the temperature measured before the run. However, if the temperature for the calculation is changed by $1 \mathrm{~K}$ there is already an offset of about $0.007 \mathrm{~s}$ between the calculated and the measured trace after 6 seconds of the tunnel run, which is about twice the accuracy of the fit. This indicates that the procedure described above is a reasonable check for the measured temperature. In fact the temperatures found by this procedure varied between $T_{0}=434 \mathrm{~K}$ and $T_{0}=440 \mathrm{~K}$ while the thermocouple did not show this variation.

The calculation of pressure and temperature allows for an accurate Reynolds number determination throughout the whole run. A linear viscosity law was assumed ${ }^{17}$ with the viscosity taken as $\mu=7.66 \pm 0.005 \times$ $10^{-6} \frac{\mathrm{Ns}}{\mathrm{m}^{2}}$ at $110.4 \mathrm{~K}$. The uncertainty of the air temperature is estimated to $\pm 0.8 \%$ and the uncertainty of the determined pressure is about $\pm 0.2 \%$. The Mach number in the test section is known from previous Pitot measurements to be $M=5.8$ in noisy operation and $M=6.0$ in quiet flow with an estimated uncertainty of $\pm 2 \%$. This enables calculating the Reynolds number in the test section with an uncertainty of about $\pm 4 \%$.

When operated quietly, the nozzle-wall boundary layer remains laminar producing free-stream noise levels ${ }^{\mathrm{a}}$ on the order of $0.05 \%$. When operated conventionally, the nozzle-wall boundary layer is turbulent and the noise level increases to about $3 \%$.

\section{III.B. Braunschweig experiments}

In Braunschweig the driver tube pressure is measured before each run with an accuracy of $\pm 1 \%$. The ratio of initial pressure, $P_{0}$, to the total pressure after the expansion, $P_{1 t}$, is well known from a large number of previous measurements $\left(P_{0} / P_{1 t}=0.9327 \pm 0.0007\right)$. The driver-tube temperature is measured during each run by two fast thermocouples. These penetrate into the flow on the upper and lower side of the valve section and enable calculating the mean temperature of the gas during a run. However, the temperature difference between the upper and lower measurement position is as high as $30 \mathrm{~K}$ and the mean of those temperatures is not proven to be the total temperature at the height of the model in the test section. This results in an estimated uncertainty of $\pm 1 \%$ in the total temperature. The Mach number in the test section has been

\footnotetext{
a Noise levels are defined here as normalized rms of Pitot pressures: $\frac{\sqrt{\overline{\left(\overline{p_{\text {pit }}}-p_{\text {pit }}\right)^{2}}}}{\overline{p_{\text {pit }}}}$
} 
determined by extensive Pitot measurements and by RANS calculations of the nozzle flow. ${ }^{12}$ In the range of unit Reynolds numbers between $4-8 \times 10^{6}$ it is $M=5.85 \pm 0.03$ at the position of the pressure sensors. This enables calculation of the Reynolds number in the test section with an uncertainty of about $\pm 2 \%$.

The nozzle-wall boundary layer in the tunnel is always turbulent. The noise level is between $1 \%$ and $1.5 \%$ depending on the unit Reynolds number, based on preliminary measurements.

\section{Data processing}

For each tunnel run in Purdue, the oscilloscope recorded 4.5 seconds of pressure signal during the run and 0.5 seconds of reference data before the start of the tunnel. From the calculated pressure and temperature the corresponding trace of the Reynolds number was calculated throughout the run. For certain Reynolds numbers at different times during each run a period of $0.4 \mathrm{~s}$ (1600000 samples) was extracted. This period was divided into overlapping windows with 2000 samples each. The offset between the windows was 200 samples giving about 8000 windows. These were multiplied with a normalized Blackman window and Fourier transformed. The absolute values of the complex-conjugate amplitudes were added and averaged over all 8000 windows. In the same way an 0.35 -s period of the data measured directly before the start of the tunnel was processed, giving only 7000 windows. The power spectra of both transforms were subtracted. Thereby the uncorrelated electronic background noise and the permanent spectrum of disturbances from the ambient electromagnetic environment was subtracted from the measured signals. For some low-noise experimental data the power spectral subtraction of background noise yielded negative amplitude values at some frequencies. These values were set to zero. It is not clear whether this preliminary method of correcting the noise is the best or most appropriate one.

The data processing for the experiments in Braunschweig was essentially the same but due to the short run time of the tunnel only a period of $60 \mathrm{~ms}$ (180000 samples) was evaluated from each run. The window size for the FFTs was chosen as 1500 samples each.

The second-mode amplification rates $-\alpha_{i}$ were calculated from

$$
\begin{array}{ll}
-\alpha_{i}=\frac{\ln \left(\frac{\hat{p}_{2}}{\hat{p}_{1}}\right)}{s_{2}-s_{1}} & \text { if } \quad \hat{p}_{1}>0 \text { and } \hat{\mathrm{p}}_{2}>0 \\
-\alpha_{i}=0 & \text { else }
\end{array}
$$

with $\hat{p}_{1}$ and $\hat{p}_{2}$ being the pressure amplitudes after noise subtraction at two consecutive sensors and $s_{2}-s_{1}$ being the surface distance between those sensors $(25.2 \mathrm{~mm})$.

\section{Stability Computations}

Stability computations using linear stability theory (LST) were made in order to compare the resulting local amplification rates to the measured results. The computations were based on both full Navier-Stokes solutions of the boundary layer flow and on similarity solutions for the compressible boundary layer. The computation methods are described here briefly.

\section{V.A. Computations based on Navier-Stokes solutions}

Laminar mean flows for the test cases were generated using an optimized 2D/axisymmetric mean flow solver based on the implicit Data-Parallel Line Relaxation (DPLR) method ${ }^{18}$ which is provided with the stability code STABL. ${ }^{19}$ The solver produces second-order accurate laminar flow solutions with low dissipation and shock capturing. For each of the simulations, $360 \times 360$ point structured grids were generated with clustering both at the body surface and at the nose.

The flow for these cases was considered to be a non-reacting mixture of $76.7 \% \mathrm{~N}_{2}$ and $23.3 \% \mathrm{O}_{2}$ by mass. Free-stream conditions were obtained by applying the isentropic flow relations from the stagnation conditions to the specified free-stream Mach numbers. Mack's viscosity model was used for the gas mixture. ${ }^{5}$

The LST analysis of the cases was performed using the PSE-Chem code which is distributed as a part of the STABL suite. ${ }^{19}$ For the LST analysis, a parallel flow assumption was made by neglecting derivatives of mean flow quantities in the direction of the computational coordinate along the body. Spatial amplification rates of disturbances were found for given disturbance frequencies and surface locations. 


\section{V.B. Computations based on similarity solutions}

Alternatively, a simplified approach has been used to compute the basic flow; this neglects the transverse curvature of the cone and the interaction of the boundary layer with the shock wave. ${ }^{20}$ Based on these assumptions the boundary layer on a sharp cone at zero angle of attack in supersonic flow can be obtained from a flat-plate boundary layer at the same edge Mach number and temperature using the transformation of Mangler and Stepanov. ${ }^{21}$ The flat-plate boundary layer was calculated from self-similar zero pressuregradient planar compressible boundary-layer equations for a calorically perfect gas. For the temperature dependence of the dynamic viscosity a modified version of the Sutherland law according to Mack ${ }^{5}$ was used. The Prandtl number was assumed to be constant and set to $\mathrm{Pr}=0.72$. An isothermal wall temperature of $315 \mathrm{~K}$ was chosen. The boundary-layer edge conditions were derived from the free-stream conditions of the experiment using the shock relations and the inviscid solution for axisymmetric supersonic conical flow. ${ }^{22}$

For the instability analysis of this self-similar boundary-layer flow the linear version of the NOLOT/PSE $\operatorname{code}^{23}$ was used. The NOLOT/PSE code was developed in cooperation between DLR and FOI and solves compressible parabolized stability equations (PSE) formulated in curvilinear orthogonal coordinates for a thermally ideal gas. Alternatively, the code can also be used as a classical local stability analysis tool. In the latter case an eighth-order system of ordinary differential equations is solved assuming a locally parallel flow. A more detailed description of the NOLOT/PSE code was reported in [24].

\section{V.C. Computations}

Two reference cases were defined for the computations as listed in Table 1. The table also gives edge quantities that have been used to plot normalized results. For the calculations, the wall temperature was

Table 1. Conditions chosen for stability analysis of $7^{\circ}$ cone boundary layer and values for normalization

\begin{tabular}{ccccccc} 
& $\mathrm{M}$ & $P_{t}[\mathrm{psia}]$ & $T_{t}[\mathrm{~K}]$ & $T_{\text {wall }}[\mathrm{K}]$ & $R e_{e} / s[1 / m]$ & $U_{e}[\mathrm{~m} / \mathrm{s}]$ \\
\hline case1 & 6.0 & 110.6 & 418 & 315 & $10.45 \times 10^{6}$ & 846 \\
case2 & 5.8 & 89.3 & 415 & 315 & $9.17 \times 10^{6}$ & 839
\end{tabular}

chosen to be slightly more than ambient temperature. Successive tunnel runs over the course of a day may have heated the model more, but temperature measurements have not been taken. A comparative stability calculation of case 1 but with $325 \mathrm{~K}$ model temperature resulted in only small deviations from the results shown here. Only 2D waves have been considered for the stability calculations. As a representative result Fig. 5 shows the amplification rates calculated from the similarity solutions of case1. The normalized plot in Fig. 5b shows that the frequency of the second mode scales well with $U_{e} \sqrt{R e_{e}} / s$. This will be used for comparison of the experimental results at different unit Reynolds numbers in the following section.

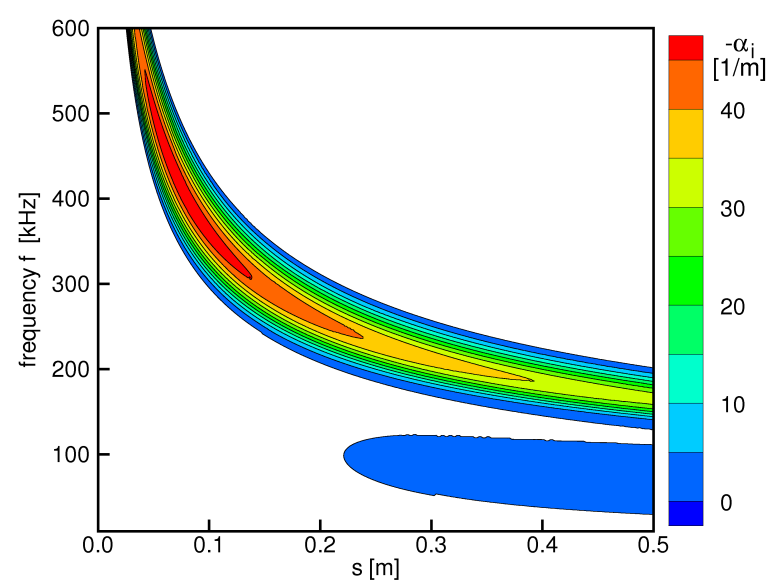

(a) Stability diagram

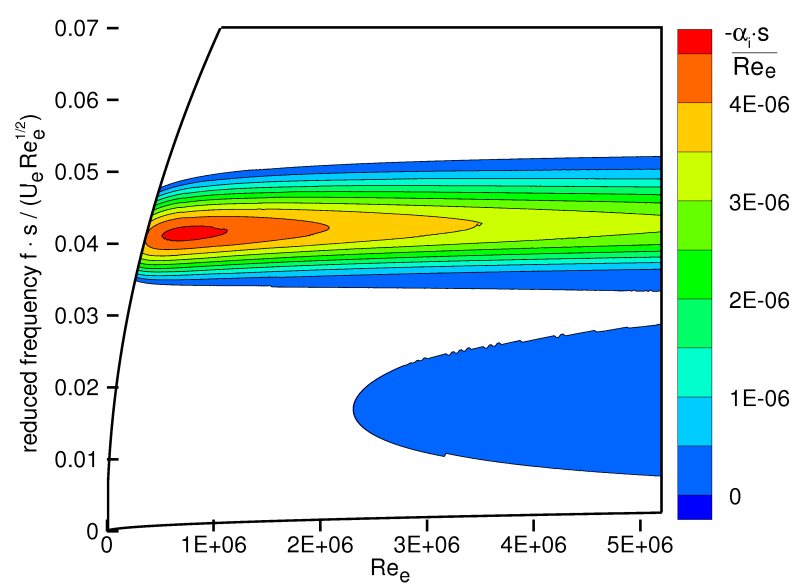

(b) Stability diagram non-dimensional

Figure 5. LST results based on similarity solution with appropriate non-dimensionalization (case1) 


\section{Results}

\section{VI.A. Pressure-Fluctuation Spectra}

Figure 6 shows samples of pressure-fluctuation spectra at three consecutive sensors. The data in Figure 6a was collected at Purdue under quiet flow at a 89.3 psia driver-tube pressure. This was the lower limit of the pressure range where the second mode could be detected under quiet flow. Note that the amplitude of the fluctuations at that pressure is close to the limit of the available resolution $\left(10^{-6} \mathrm{psi}\right.$ at $\left.16 \mathrm{bit}\right)$. Therefore the signal-to-noise ratio is very small and only the large number of averaged FFTs (8000 windows) brings out the 2 nd mode peaks. Also note that the resolution of the pressure sensors is specified as $10^{-3} \mathrm{psi}$ in the manufacturer's datasheet. Moreover the diameter of the sensing surface $(3.18 \mathrm{~mm})$ is larger than half

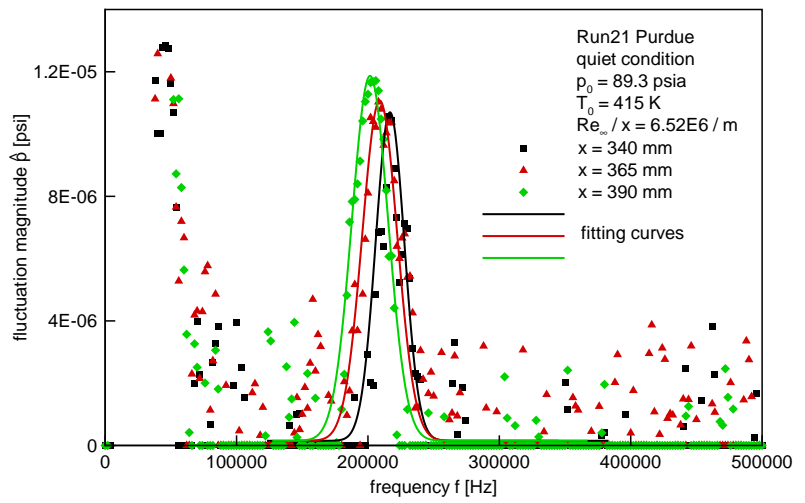

(a) measurement and curve fit

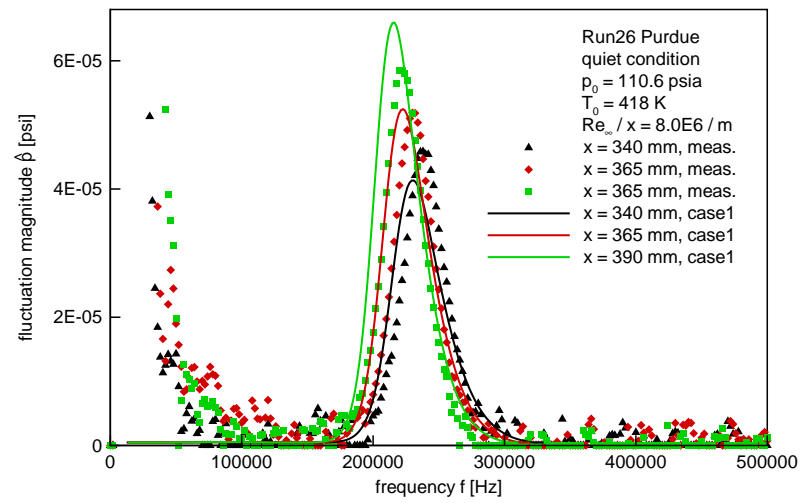

(b) measurement and calculation

Figure 6. Spectra of pressure fluctuations measured in quiet Mach-6 flow

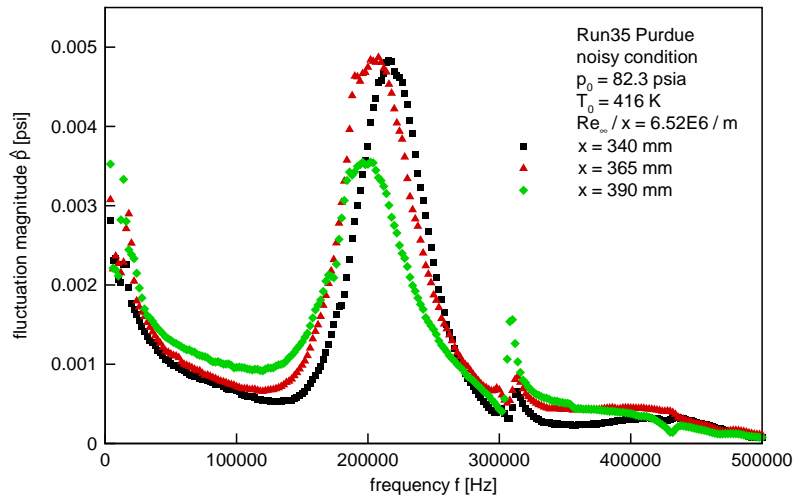

(a) noisy condition Purdue

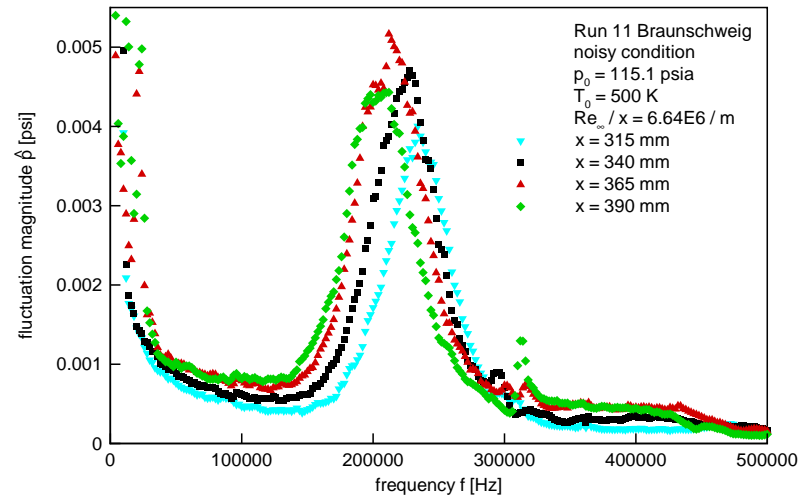

(b) noisy condition Braunschweig

Figure 7. Spectra of pressure fluctuations measured in noisy Mach-5.8 flow

the wavelength of the second-mode waves, where half the wavelength can be approximated by the boundary layer thickness of $\simeq 2 \mathrm{~mm}$. For these reasons, the absolute amplitudes given in the figure are questionable and have to be understood as some uncertain nonlinear function of the actual fluctuation amplitude at a certain frequency. However, the measured amplitude is assumed to be linearly proportional to the mean amplitude of the fluctuations when amplification rates are calculated in this paper. The spectrum shown in Fig. 6b was measured at 110.6 psia driver-tube pressure and shows a second-mode amplitude that is an order of magnitude higher. The figure also shows integrated amplification rates from the linear stability calculation of case1 based on the similarity solution. The calculated amplitudes are scaled to match the amplitude at the second sensor position. The measured amplification seems to be somewhat higher than the calculation. The calculated frequencies are lower than those measured, by about $8 \mathrm{kHz}$. This frequency shift may be due to the suspected angle of attack discussed in section II.E. However, the bandwidth of amplified frequencies is almost the same in the measurement and the calculation. 
Figure 7 shows spectra in noisy flow measured at the same Reynolds number as in Fig. 6a, at similar stagnation conditions. In noisy flow the measured pressure amplitudes are about 450 times higher. The second-mode peaks in noisy flow are much broader and first harmonics can be detected. The small peak at about $310 \mathrm{kHz}$ is thought to be an effect of the sensors although this is far below the resonant frequency claimed by the manufacturer. At this Reynolds number in noisy flow the boundary layer appears to be transitional, since the second mode amplitude growth stagnates and reverses between the second and third stream-wise sensor. Note that the results in the Braunschweig tunnel show the same behavior at nearly the same Reynolds number and wave amplitude (Fig. 7b). Since no data was collected at Purdue at lower unit Reynolds numbers than shown in Fig. 7, we can compare data at the noisy condition only, as described with the Braunschweig experiments in the following section.

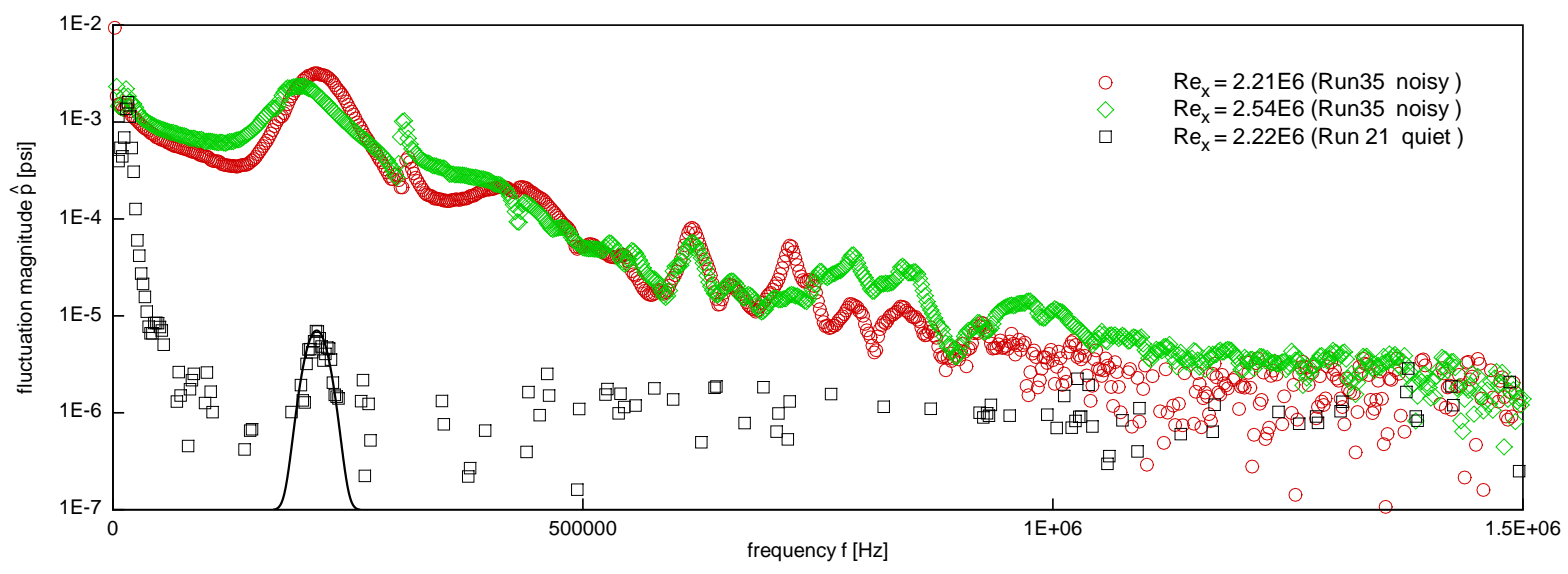

Figure 8. Spectra of pressure fluctuations measured in noisy and quiet flow at same Reynolds numbers

Figure 8 plots the data of Figs. 6a and $7 \mathrm{a}$ together using a logarithmic scale. The logarithmic plot shows that in the transitional boundary layer both the lower and higher frequency portions of the spectrum are starting to fill in. However, it is not yet possible to determine how much of the difference in spectra is due to nonlinear amplification and breakdown of the waves, and how much is due to the difference in the spectrum of the free-stream noise.

Since under quiet flow at this pressure the second mode is just becoming detectable, the measurement technique does not permit comparing linear amplification rates at the same Reynolds number in quiet and noisy hypersonic flow. It will be necessary to use controlled perturbations in the quiet-flow boundary layer to make such a comparison.

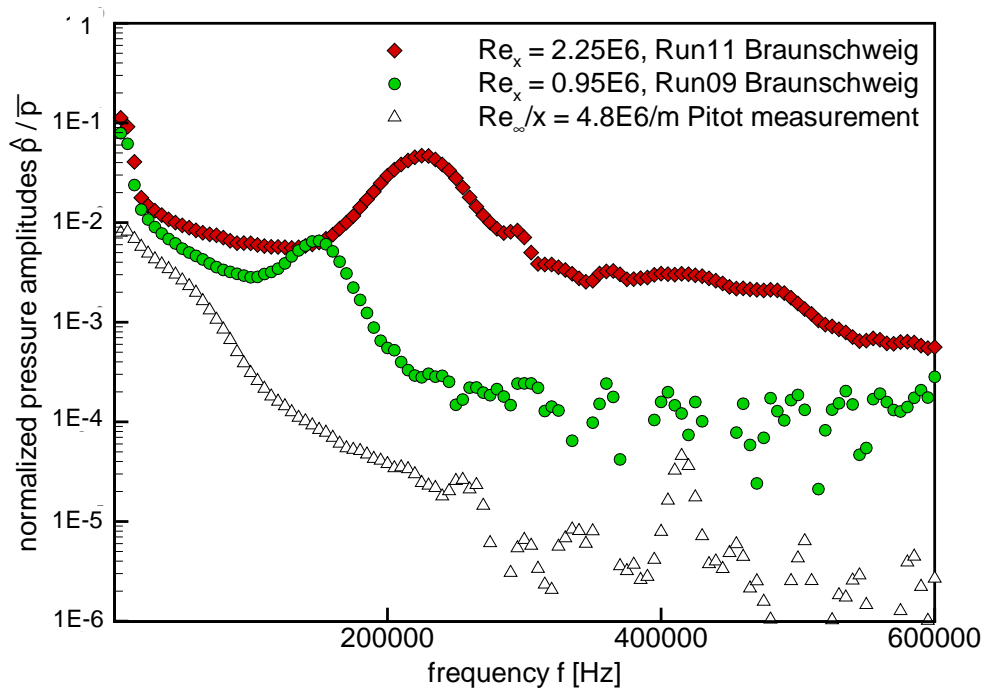

Figure 9. Surface pressure fluctuations normalized by calculated edge pressures at two different Reynolds numbers compared to normalized Pitot pressure fluctuations measured in the Braunschweig tunnel 
Figure 9 shows normalized pressure spectra measured at the cone surface at two different Reynolds numbers compared to a measured Pitot pressure spectrum. The surface pressure spectrum at the higher Reynolds number is the one from Figure 7b at $x=340 \mathrm{~mm}$ which is just before stagnation of the secondmode growth. Note, that the Pitot spectrum was measured with an absolute pressure sensor providing the actual mean pressure for normalization. Whereas the surface pressure spectra were normalized with the edge pressure calculated from inviscid conical flow solution at $\mathrm{M}=5.8$ for the measured stagnation pressure. Therefore the normalization of the surface pressure spectra is somewhat uncertain. However, the normalized surface pressure fluctuations are by almost a factor 2 higher than the Pitot fluctuations already at low frequencies. The reason for that difference is not clear. An effect of receptivity or an early amplification of disturbances within the cone boundary-layer can be suspected.

Often rms values of pressure measurements are used to compare noise levels, although this quantity does not give any information on the spectral distribution of the fluctuations. However, in order to give some idea of how much the rms of the fluctuations rises before transition occurs it may be of interest to provide the rms values for the three measurements shown in Figure 9; $\operatorname{rms}(\triangle)=1.4 \% ; \operatorname{rms}(\bigcirc)=6.7 \% ; \operatorname{rms}(\diamond)=$ $13 \%$.

\section{VI.B. Amplification rates}

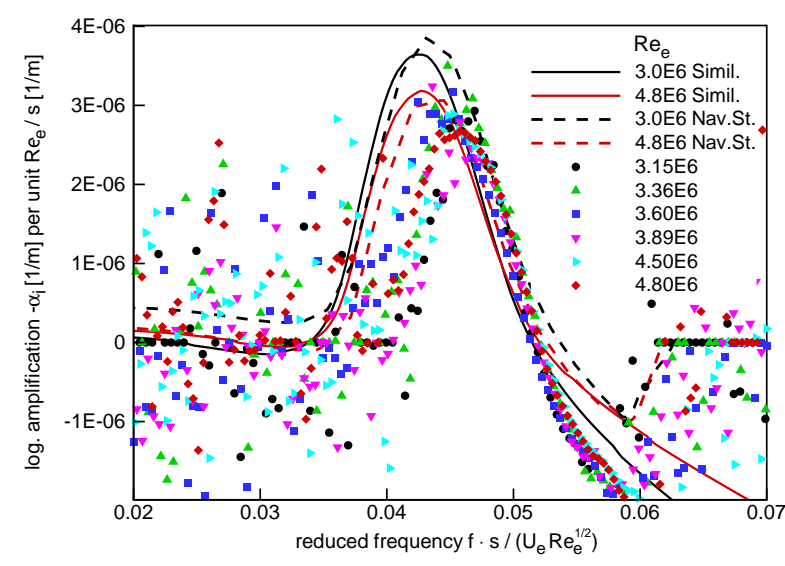

(a) quiet condition Purdue

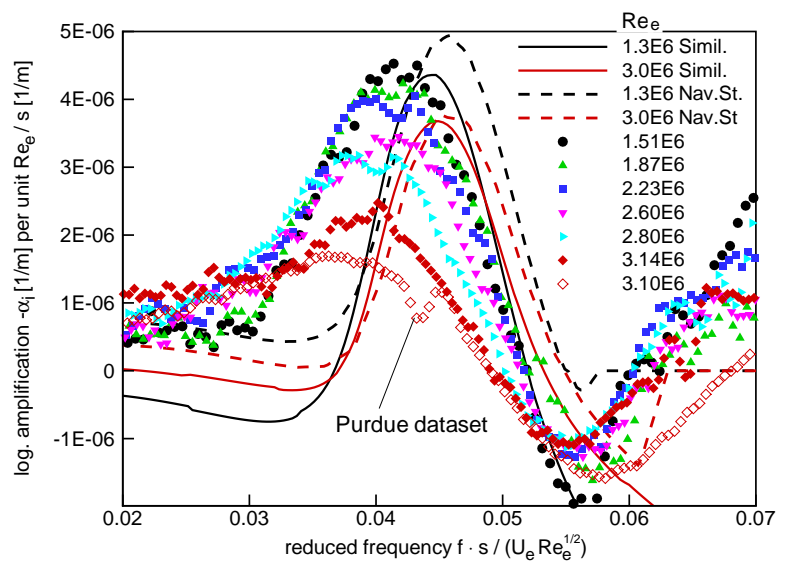

(b) noisy condition Braunschweig

Figure 10. Amplification rates as calculated from measured pressure amplitudes compared to LST values based on Navier-Stokes solution and on similarity solution

Fig. 10 shows the logarithmic ratio of the amplitudes normalized by the unit edge Reynolds number vs. the reduced frequency, in both quiet flow (a) and noisy flow (b). The results shown for the quiet cases are averages over two to four datasets from different runs at the same Reynolds number, whereas the results shown for noisy data are from single tunnel runs. The scattering of the data in quiet flow at the low frequency edge of the amplified spectrum is caused by taking a ratio of weak signals with very low signal-to-noise ratio. Nevertheless, the trend of that data shows that the measured quiet-flow bandwidth for the amplified waves is narrower than predicted by the calculations. Moreover, the amplification rates of the quiet cases are smaller than predicted by linear stability theory. However, both shortcomings may be due to the suspected angle of attack (see section II.E), or possibly to the relatively large sensor size.

The measured maximum amplification rates in noisy flow agree well with calculations at the lower Reynolds numbers. However, even at the smallest Reynolds numbers measured, the measurements show amplification at lower frequencies than the computations. For edge Reynolds numbers higher than $2.2 \times 10^{6}$ the maximum amplification rates rapidly decrease. This is in accordance with Stetson's observations ${ }^{25}$ who reported this behaviour at Mach 8 for $\sqrt{R e_{e}}>1400$. Stetson also reported a growth of harmonics of the second mode at higher Reynolds numbers which he suspected was due to nonlinear effects. But, looking at the amplification rates of higher frequencies in Fig. 11 shows that for all Reynolds numbers measured in noisy flow the amplification rate of the harmonics is the same as the fundamental amplification. Hence, it seems that this is not necessarily an indicator for nonlinear growth, but just a result of the pressure signature on a sensor's surface being more complex than a pure sine wave. Nevertheless, comparing the amplification rates 


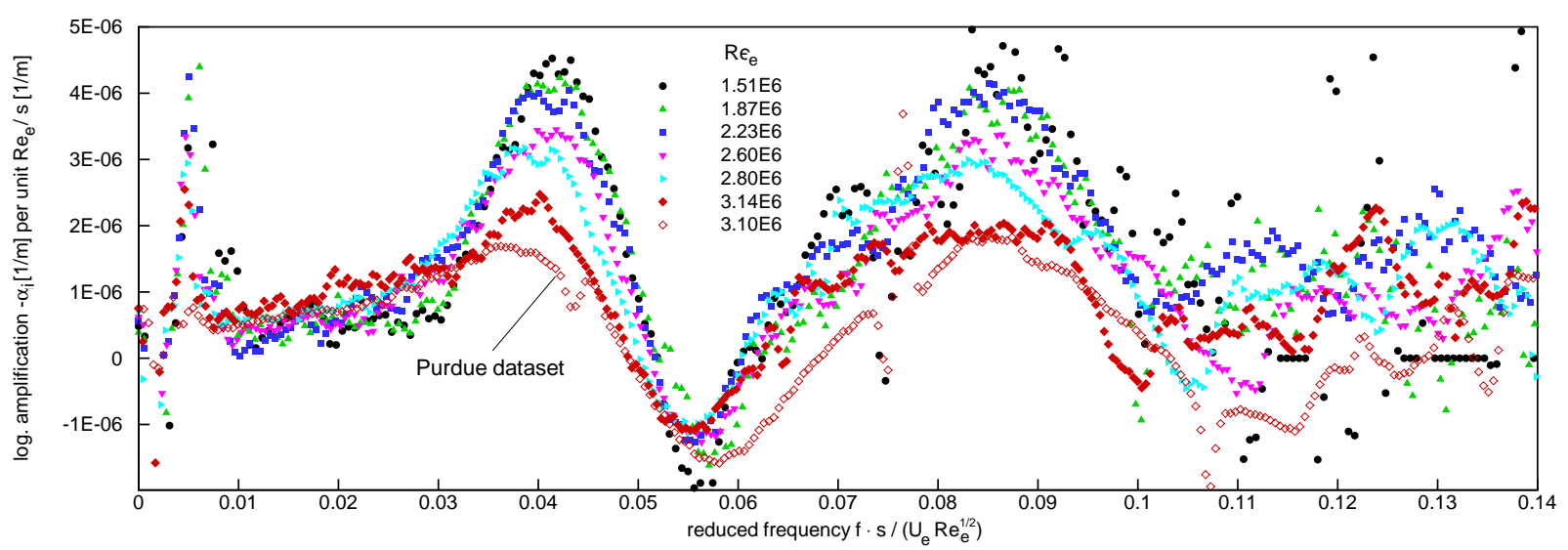

Figure 11. Amplification rates as calculated from measured pressure amplitudes, extended spectrum from Fig. 10b

measured in noisy flow to those measured in quiet flow there is a significant difference in the bandwidth of amplified frequencies which may be due to nonlinear effects even at the lowest measured Reynolds numbers. For the given cone, the lowest feasible Reynolds number was limited by the operational range of the facility in Braunschweig. At those conditions in noisy flow the second mode signal was still 50 times larger than the weakest second-mode wave that was detectable under quiet flow. In Stetson's ${ }^{26}$ experiments with hot wires at Mach 8 , the second-mode was first detectable at $R e_{e} \approx 1.2 \times 10^{6}$, which is close to the smallest Reynolds number analyzed here in noisy flow.

\section{Conclusion}

The use of high frequency surface-pressure sensors for measuring pressure fluctuations caused by secondmode boundary layer instabilities has been proven to be very effective even in quiet hypersonic flow. The advantages of this technique over hot-wire measurements for determining stream-wise amplification rates are:

1. No downstream influence of the sensors is apparent and therefore stream-wise arrays of sensors can be used to easily access spatial amplification rates for instabilities.

2. This is an easy-to-use off-the-shelf measurement technology at comparatively low cost.

3. The sensors used are very resistant to the harsh environment typical of hypersonic facilities.

4. The high frequency response of the sensors allows for measurements up to the $1 \mathrm{MHz}$ range.

5. The sensitivity for detecting the second mode in terms of signal-to-noise ratio may be an order of magnitude better than in the hot-wire experiments by Stetson et al., although Stetson et al. used completely different data acquisition systems.

These very promising preliminary results suggest that the limited spatial resolution of the surface-pressure sensors might be alleviated through careful comparison to spatial averages of the computational results.

The second-mode amplitudes under noisy conditions were 450 times higher than under quiet flow at the same Reynolds number. This ratio of the second-mode amplitude seems to be much larger than the ratio of the broadband Pitot fluctuations, which was about 50-100, perhaps due to the spectral content of the free-stream noise and also to receptivity effects.

Under noisy conditions, the second-mode amplitudes began to decrease at about the same Reynolds number in both the Purdue and Braunschweig tunnels, which suggests that results in the two tunnels can be compared to enable the study of nonlinear effects. Under quiet flow, at this same Reynolds number, the instability amplitudes just began to rise from the measurement noise. Therefore it was not possible to make a direct comparison of second mode amplification rates in noisy and quiet flow.

Measured maximum amplification rates were in good agreement with those calculated using linear stability codes. In addition, the bandwidth of amplified frequencies was in good agreement under quiet flow. The 
remaining differences are thought to be due to a slight angle of attack in the quiet experiments. However, under noisy conditions the computed bandwith of amplified frequencies was more broad than the measurements, for all Reynolds numbers down to $R e_{e}=1.5 \times 10^{6}$. The measured noisy-flow maximum amplification rates were in good agreement with calculations below $R e_{e}=2.2 \times 10^{6}$, as in Stetson's observations at Mach 8 .

Future measurements should check the angle of attack more carefully by comparing the frequencies of the most-unstable wave on opposite sides of the cone. It would also be very interesting to compare measurements of the instability waves using surface-pressure sensors to measurements using a hot wire or laser differential interferometer. A series of measurements with the surface pressure sensors under different conditions might be combined with computations and measurements using other instrumentation, in order to determine the effect of the size of the pressure sensor. The pressure sensors can then be used in several tunnels to compare 2nd-mode wave growth under conditions where hot wires will not survive.

\section{A. Appendix}

Figure $4 \mathrm{a}$ shows an idealized characteristic diagram of the flow in the tube shortly after the start of the tunnel. The expansion wave starting at the nozzle at $t=0$ is modeled by two characteristic lines corresponding to the head and the tail of the wave $\mathrm{b}^{\mathrm{b}}$. The wave is reflected at the boundaries after each pass through the tube. The boundary conditions at the ends of the tube are found as follows: At the throat an unknown and time dependent critical mass flux per area $a^{*} \rho^{*}$ can be assumed with $a^{*}$ being the velocity of sound at critical condition and $\rho^{*}$ being the critical density. When $A^{*}$ is the combined throat area for the main flow and the bleed slot, the corresponding mass flux per area at the tube diameter $A_{t}$ is $\rho u=\rho^{*} a^{*} \frac{A^{*}}{A_{t}}$, with $\rho$ and $u$ being the area-averaged density and velocity at diameter $A_{t}$. The ratio of the mass fluxes per area is related to the flow Mach number $M_{t}$ by the equation for a one-dimensional stationary isentropic expansion:

$$
\frac{\rho u}{\rho^{*} a^{*}}=M_{t}\left(\frac{2+(\gamma-1) M_{t}^{2}}{\gamma+1}\right)^{-\frac{\gamma+1}{2(\gamma-1)}}=\frac{A^{*}}{A_{t}} .
$$

Therefore the Mach number in the tube is a function only of the area ratio and independent of the state of the fluid in the tube. Hence, the boundary conditions of the characteristics for $t>0$ become: $u=M_{t} \cdot a$ at the right end of the tube, which is at $x=0$, assuming the contraction length is small compared to the tube length, and $u=0$ at the upstream end where $x=-l$, with $l$ being the tube length. With these assumptions the state of the gas in areas $I$ to III (and after all subsequent passes of the expansion wave) can be calculated as a function of the tube Mach number by the following procedure. However, the exact tube Mach number is not known in advance due to the unknown effect of the boundary layer's displacement thickness on the effective tube diameter. Therefore the Mach number has to be determined from the measured pressure drop after each reflection of the expansion wave.

The head of the expansion wave travels with the velocity of sound $a_{0}$ into the gas. So the slope of the characteristic line in the diagram is $\frac{d x}{d t}=-a_{0}$ before its reflection at $A_{1}$ (Fig. 4a). At point $B_{1}$ the head of the reflected expansion wave intersects the tail of the incident expansion wave. Between $A_{1}$ and $B_{1}$ its slope changes to $a_{I}\left(1+M_{t}\right)$, according to the state of the gas in area $I$, fulfilling the downstream boundary condition. The conditions in area $I$ can be calculated by integrating the compatibility equation for the $C_{+}$ characteristic from $A_{1}$ to $B_{1}$

$$
-\int_{0}^{a_{I} M_{t}} d u=\frac{\sqrt{\gamma R}}{\gamma-1} \int_{0}^{I} \frac{d T}{\sqrt{T}}
$$

which gives

$$
-a_{I} M_{t}=\frac{2 a_{0}}{\gamma-1}\left[\left(\frac{T_{I}}{T_{0}}\right)^{\frac{1}{2}}-1\right] .
$$

and rearranging with $\frac{a_{I}}{a_{0}}=\left(\frac{T_{I}}{T_{0}}\right)^{\frac{1}{2}} \operatorname{gives}^{27}$

$$
\frac{T_{I}}{T_{0}}=\left(1+\frac{\gamma-1}{2} M_{t}\right)^{-2} .
$$

\footnotetext{
${ }^{\mathrm{b}}$ Using more than two characteristic lines would complicate the calculation. However, the very low Mach number in the driver tube results in weak waves that are well captured with only two characteristic lines.
} 
The pressure and density ratios can be calculated from Eq. (4) using isentropic relations. ${ }^{22,27}$ The initial slope of the tail of the expansion wave is $a_{I}\left(M_{t}-1\right)$ before its reflection in $C_{1}$ and $a_{I I}$ thereafter. The state of the gas in area $I I$ is calculated by integrating the compatibility equation for the $C_{-}$characteristic from $B_{1}$ to $C_{1}$

$$
\int_{a_{I} M_{t}}^{0} d u=\frac{\sqrt{\gamma R}}{\gamma-1} \int_{I}^{I I} \frac{d T}{\sqrt{T}} .
$$

Performing the integration and using Eqs. (3) and (4) gives after rearrangement

$$
\frac{T_{I I}}{T_{0}}=\left[\frac{2-(\gamma-1) M_{t}}{2+(\gamma-1) M_{t}}\right]^{2} .
$$

The state in area $I I I$ can be found by integrating from $B_{2}$ to $C_{2}$. Since the fluid in area $I I$ is at rest its condition can be regarded as the new initial state $\left(T_{I I}=T_{0^{\prime}}\right)$. Then the integration from $B_{2}$ to $C_{2}$ is similar to that from $A_{1}$ to $A_{2}$ and the relations for all subsequent reflections are given by Eqs.(4) and (6). So finally the static temperature at the contraction after time $t_{C_{2 n}}$ with $n=1,2,3 \ldots$ changes to

$$
T_{2 n+1}=T_{0}\left(1+\frac{\gamma-1}{2} M_{t}\right)^{-2}\left[\frac{2-(\gamma-1) M_{t}}{2+(\gamma-1) M_{t}}\right]^{2 n} .
$$

The time needed for the head of the wave to travel forth and back in the tube can be calculated from the slope of the characteristic lines

$$
t_{A_{2}}=\frac{l}{a_{0}}+\frac{l}{a_{1}\left(1+M_{t}\right)}=\frac{l}{a_{0}}\left(\frac{2+\frac{\gamma+1}{2} M_{t}}{1+M_{t}}\right) .
$$

The time between the arrival of the head and tail at $x=0$ is

$$
t_{B_{2}}-t_{A_{2}}=\frac{l}{a_{0}}\left[\frac{2+(\gamma-1) M_{t}}{2-(\gamma-1) M_{t}}-1\right] .
$$

Adding the traveling times of the head for subsequent reflections using Eq. (6) gives

$$
t_{A_{2 n}}=\frac{l}{a_{0}}\left(\frac{2+\frac{\gamma+1}{2} M_{t}}{1+M_{t}}\right)\left[1+\sum_{k=1}^{n-1}\left[\frac{2+(\gamma-1) M_{t}}{2-(\gamma-1) M_{t}}\right]^{k}\right] .
$$

Accordingly, the time lag between the head and the tail of the wave after several reflections becomes

$$
t_{C_{2 n}}-t_{A_{2 n}}=\frac{l}{a_{0}}\left[\frac{2+(\gamma-1) M_{t}}{2-(\gamma-1) M_{t}}+M_{t} \frac{2+(\gamma-1) M_{t}}{1-M_{t}^{2}}-1\right] \cdot\left[1+\sum_{k=1}^{n-1}\left[\frac{2+(\gamma-1) M_{t}}{2-(\gamma-1) M_{t}}\right]^{k}\right] .
$$

Note that for the calculation of these times, due to the modeling by only two characteristic lines, a step change of the traveling speed was assumed at the boundaries rather than a continuous change within the overlapping areas ABC. So the above formulas will underestimate the travel time for the head and overestimate that for the tail of the wave. Therefore the exact path of the expansion wave will be somewhere between the calculated characteristics. Hence, the uncertainty of the path is given by somewhat less than half of the distance between head and tail. For the given tube with a length of 125.5 feetc and a Mach number $M_{t}=0.0046$ at temperature $T_{0}=433 \mathrm{~K}$ the time lag between head and tail can be calculated for $\mathrm{n}=15$ from Eq. (10). It is $t_{C_{30}}-t_{A_{30}}=0.015 \mathrm{~s}$ which is about $0.5 \%$ of the overall traveling time at that point.

\section{Acknowledgments}

The first author would like to thank Professor Schneider's research group for their great support during his 3 month stay at Purdue. Dr. K. Fujii of JAXA was kind enough to give full information regarding the sensors used in his experiments and also by the present authors. Operating costs for the Purdue tunnel were funded by AFOSR under grant FA9550-06-1-0182.

cThe length of the tube is 122.5 feet and the contraction has a length of 40 inches. The last 4 inches of the contraction have been neglected in the calculation, since the sudden rise of the Mach number will cause the upstream information to fade somewhere close to the throat. 


\section{References}

${ }^{1}$ Schlichting, H. and Gersten, K., Boundary Layer Theory, Springer, Berlin, 8th ed., 2000.

${ }^{2}$ Schneider, S. P., "Hypersonic laminar-turbulent transition on circular cones and scramjet forebodies," Progress in Aerospace Sciences, Vol. 40, 2004, pp. 1-50.

${ }^{3}$ Pate, S. R. and Schueler, C. J., "Radiated Aerodynamic Noise Effects on Boundary-Layer Transition in Supersonic and Hypersonic Wind Tunnels," AIAA Jounal, Vol. 7, No. 3, 1969, pp. 450-457.

${ }^{4}$ Beckwith, I. E. and Miller III, C. G., "Aerothermodynamics and transition in high-speed wind tunnels at NASA Langley," Annu. Rev. Fluid Mech., Vol. 22, 1990, pp. 419-439.

${ }^{5}$ Mack, L. M., "Boundary-Layer Linear Stability Theory," Special Course on Stability and Transition of Laminar Flow, AGARD Special Course at the von Kármán Institute Rhode-Saint-Genèse, Belgium, March 26-30, 1984., AGARD Report No. 709, 1984, pp. 3-1-3-81.

${ }^{6}$ Fujii, K., "Experiment of Two-Dimensional Roughness Effect on Hypersonic Boundary-Layer Transition," J Spacecraft and Rockets, Vol. 43, 2006, pp. 731-738.

${ }^{7}$ Juliano, T. J., Swanson, E. O., and Schneider, S. P., "Transition Research and Improved Performance in the Boeing/AFOSR Mach-6 Quiet Tunnel," AIAA Paper 2007-0535, 2007.

${ }^{8}$ Schneider, S. P., "The Development of Hypersonic Quiet Tunnels," AIAA Paper 2007-4486, 2007.

${ }^{9}$ Schneider, S. P. and Juliano, T. J., "Laminar-Turbulent Transition Measurement in the Boeing/AFOSR Mach-6 Quiet Tunnel," AIAA Paper 2007-4489, 2007.

${ }^{10}$ Borg, M. P., Schneider, S. P., and Juliano, T. J., "Effect of Freestream Noise on Roughness-Induced Transition for the X-51A Forebody," AIAA Paper 2008-0592, 2008.

${ }^{11}$ Wolf, T., Estorf, M., and Radespiel, R., "Investigation of the starting process in a Ludwieg tube," Theoretical and computational Fluid Dynamics, Vol. 21, 2007, pp. 81-98.

${ }^{12}$ Estorf, M., Wolf, T., and Radespiel, R., "Experimental and numerical investigations on the operation of the Hypersonic Ludwieg Tube Braunschweig," 5th European Symposium on Aerothermodynamics for Space Vehicles, 2004.

${ }^{13}$ Estorf, M., Radespiel, R., Heine, M., and Müller-Eigner, R., "Der Hyperschallwindkanal Ludwiegrohr Braunschweig HLB," DGLR-Jahrbuch 2003, 2003, pp. 661-670.

${ }^{14}$ PCB Piezotronics, "Pressure and Force Sensors Division, Pressure Catalog," http://www.pcb.com/Linked_Documents /Pressure/PFScat.pdf, 2007.

${ }^{15}$ Estorf, M., Ortsaufgelöste Messung instationärer Wärmestromdichten in der Aerothermodynamik, Eingereichte Dissertation, Fakultät für Maschinenbau TU Braunschweig, 2007.

${ }^{16}$ Borg, M. P., Schneider, S. P., and Juliano, T. J., "Inlet Measurements and Quiet Flow Improvements in the Boeing/AFOSR Mach-6 Quiet Tunnel," AIAA Paper 2006-1317, 2006.

${ }^{17}$ Fiore, A. W., "Viscosity of Air," J Spacecraft and Rockets, Vol. 3, 1966, pp. 756-758.

${ }^{18}$ Wright, M. J., Candler, G. V., and Bose, D., "A Data-Parallel Line-Relaxation Method for the Navier-Stokes Equations," Paper 97-2046CP, AIAA, 1997.

${ }^{19}$ Johnson, H. B. and Candler, G. V., "Hypersonic Boundary Layer Stability Analysis Using PSE-Chem," Paper 2005-5023, AIAA, June 2005.

${ }^{20}$ Simen, M. and Dallmann, U., "On the instability of hypersonic flow past a pointed cone - Comparison of theoretical and experimental results at Mach 8-," DLR research report FB 92-02, 1992.

${ }^{21}$ Stewartson, K., The theory of laminar boundary layers in compressible fluids, Oxford University Press, 1964.

${ }^{22}$ Shapiro, A. H., The Dynamics and Thermodynamics of Compressible Fluid Flow, Vol. 1, The Ronald Press Company, New York, 1953

${ }^{23}$ Hein, S., Bertolotti, F. P., Simen, M., Hanifi, A., and Henningson, D., "Linear nonlocal instability analysis, - the linear NOLOT code -," Tech. Rep. DLR-IB 223-94 A56, Institut für Strömungsmechanik, DLR Göttingen, 1994.

${ }^{24}$ Rosenboom, I., Hein, S., and Dallmann, U., "Influence of Nose Bluntness on Boundary-Layer Instabilities in Hypersonic Cone Flows," AIAA Paper 99-3591, 1999.

${ }^{25}$ Stetson, K. and Kimmel, R., "On Hypersonic Boundary Layer Stability," AIAA Paper 92-0737, 1992.

${ }^{26}$ Stetson, K., Thompson, E. R., and Donaldson, C. J., "Laminar Boundary Layer Stability Experiments," AIAA Paper 83-1761, 1983.

${ }^{27}$ Becker, E., Gasdynamik, Vol. 6 in Leitfäden der angewandten Mathematik und Mechanik, B. G. Teubner, Stuttgart, 1966 . 